\title{
Distributed Path Planning of Unmanned Aerial Vehicle Communication Chain Based on Dual Decomposition
}

\author{
Xiaohua Wei $(\mathbb{D})$ and Jianliang Xu \\ School of Mechanical and Electrical Engineering, Quzhou College of Technology, Quzhou, Zhejiang 324000, China \\ Correspondence should be addressed to Xiaohua Wei; 377036471@qq.com
}

Received 29 December 2020; Revised 20 May 2021; Accepted 25 May 2021; Published 7 June 2021

Academic Editor: Wei Wang

Copyright (c) 2021 Xiaohua Wei and Jianliang Xu. This is an open access article distributed under the Creative Commons Attribution License, which permits unrestricted use, distribution, and reproduction in any medium, provided the original work is properly cited.

\begin{abstract}
Limited by the insufficiency of single UAV's load and flight time capabilities, the multi-UAV (unmanned aerial vehicle) collaboration to improve mission efficiency and expand mission functions has become the focus of current UAV theory and application research. In this paper, the research on UAV global path planning is carried out using the ant colony algorithm, and an indoor UAV path planning model based on the ant colony algorithm is constructed. In order to improve the efficiency of the algorithm, enhance the adaptability and robustness of the algorithm, a distributed path planning algorithm based on the dual decomposition UAV communication chain is proposed. This algorithm improves the basic ant colony algorithm from the aspects of path selection, pheromone update, and rollback strategy in view of the inherent shortcomings of the ant colony algorithm. In order to achieve the best performance of the algorithm, this paper analyzes each parameter in the ant colony algorithm in depth and obtains the optimal combination of parameters. The construction method of the Voronoi diagram was improved, and the method was simulated to verify that the method can obtain a Voronoi diagram path that is safer than the original method under certain time conditions. Through the principle analysis and simulation verification of the Dijkstra algorithm and the dual decomposition ant colony algorithm, it is concluded that the dual decomposition ant colony algorithm is more efficient in pathfinding. Finally, through simulation, it was verified that the dual decomposition ant colony algorithm can plan a safe and reasonable flight path for multiple UAV formation flights in an offline state and achieve offline global obstacle avoidance for multiple UAVs.
\end{abstract}

\section{Introduction}

UAV (unmanned aerial vehicle) is widely used in military warfare and people's livelihood due to its low cost, ease of operation, fearlessness, and danger [1]. Militarily, UAV can perform tasks such as battlefield reconnaissance and surveillance, ship escort, and air early warning [2]. It can also be used as bait to attract enemy firepower, or as a target aircraft for army training. In civil use, UAV can be used for aerial photography, disaster monitoring, traffic patrol, and security monitoring [3].

Algorithms are the core content of path planning problems. Excellent path planning algorithms can not only ensure the optimal global path but also accelerate the speed of path replanning when encountering unexpected situations, thereby improving the overall efficiency [4-6]. Commonly used path planning algorithms include genetic algorithm, probability map method, particle swarm algorithm, and artificial potential field method [7]. Among them, the genetic algorithm draws on the genetic laws of survival of the fittest in nature, through selection, crossover, mutation, and other means, and finally achieves an optimal solution [8]. The algorithm is simple in process, has many advantages, can perform fast and random search, and does not require high details of the problem. It has strong robustness and is easy to be combined with other algorithms. In recent years, great progress has been made in the research of UAV path planning based on genetic algorithm, but there are still some defects, such as slow convergence speed and premature fall into local optimality [9]. At the same time, because the genetic algorithm involves the selection of multiple parameters, the parameter selection is different, and the convergence effect of the 
algorithm is different. If these parameters are not properly selected, the optimal solution will not be obtained [10]. For example, the selection of fitness function not only needs to consider the algebraic value of the flight path but also needs to consider the path feasibility [11]. Therefore, if it is not selected properly, it may not get the global optimal and converge to the local optimal.

Related scholars have proposed a double genetic algorithm mechanism [12]. The method achieves two evolutionary goals based on different fitness functions and plans paths for static and dynamic threats in the environment, which can find the optimal path while avoiding threats [13]. The researchers applied genetic algorithms to the solution of path planning problems and used polar coordinates to describe the location of path points and threats, which improved the efficiency of path planning [14]. Relevant scholars solved the problem of avoiding obstacles for robots by manually setting gravitational fields and repulsive fields [15]. The basic idea of this method is to abstract UAV motion in the mission environment as a kind of motion in a man-made field, construct a gravitational field for the target point, so that it produces "gravitation" for UAV, and the threat area has a repulsive field [16]. It produces "repulsive force" and guides the UAV's flight through the combined force. This method can realize fast control, so it is widely used for real-time motion control. The planned path is safe and smooth, but when the threat is close to the target area, because the repulsive force is greater than the gravitational force, the UAV will not be able to reach the target position, and this method may fall into local optimum [17]. Relevant scholars generate path points, construct a connected graph in free space, and convert the solution space of the path planning problem into a topological space, so that the complexity of the problem is independent of the complexity of the environment and the dimension of the planning space [18]. The disadvantage of this method is that when the obstacles in the planning space are dense or there are narrow passages, the efficiency of the method will become low $[19,20]$. In addition, because this method randomly samples the signpost nodes when constructing the connected graph, it is easy to cause the final search path to deviate from the optimal.

Depending on the degree of master of environmental information, path planning can be divided into global path planning and local path planning, environmental information offline completed entirely known cases before the flight path planning is called the global path planning, environmental information is not completely known. Before global path planning, it is necessary to master comprehensive environmental information and carry out path planning according to all information in the map. Local path planning does not require known comprehensive environmental information. Instead, sensors collect environmental information in real time to determine the distribution of obstacles around the aircraft and carry out path planning in the local range. The research objects of path planning include robots, ground vehicles, underwater submarines, and aircraft. The path planning of aircraft is usually called track planning, while in the field of indoor microunmanned aerial vehicle autonomous navigation, the flight planning process is usually called path planning rather than track planning.

In this paper, we use the ant colony algorithm to study UAV global path planning and carry out in-depth and detailed theoretical research and analysis on the key problems in the ant colony algorithm. In order to solve the problem that the ant colony algorithm has a slower initial solution and is easy to fall into local optimization, a UAV global path planning algorithm is proposed. And we optimize the selection of important parameters in the algorithm, so that the algorithm has the best results in terms of optimization ability and algorithm efficiency. On the basis of identifying obstacle modalities in the global environment, the safety circle is delimited for the obstacle, and the safety circle area is set as the dangerous area. In the process of generating the Voronoi diagram path diagram, it focuses on the improved method of finding the delta center of the duality generation method after generating Delaunay triangulation elements, and the method was simulated to verify that the method can obtain a safer Voronoi diagram path. Through simulation verification, it is concluded that the dual decomposition ant colony algorithm in the global path search is more efficient than the Dijkstra algorithm in time and space. For this topic, the dual decomposition ant colony algorithm is selected as the global obstacle avoidance method.

The organizational structure of this paper is as follows: in the second part, the related theories and key methods are introduced. In the third part, the global path planning algorithm of UAV based on the ant colony algorithm is designed. In the fourth part, the methods proposed in this paper are simulated, and the results are analyzed and discussed. Finally, the full text is summarized.

\section{Related Theories and Key Methods}

2.1. Dual Optimization Theory. The optimization theory in mathematics is to discuss what kind of plan is the best and how to find the best plan among many plans. The dual optimization theory is an important branch of optimization theory. The following is a brief introduction to the dual optimization theory to provide a basis for the research discussion.

The general optimization problem is as follows:

$$
\begin{array}{cc}
\min & f_{0}(x) \\
f_{i}(x)<, i=1,2, \cdots m & h_{i}(x)=0, i=1,2, \cdots, p
\end{array} .
$$

The basic idea of Lagrange duality is to extend the objective function by weighting the constraint function. The Lagrange function of the optimization problem is defined as follows:

$$
L(x, v, \lambda)=\sum_{i=1}^{p} v_{i} h_{i}(x)+\sum_{i=1}^{m} f_{i} \lambda_{i}(x)+f_{0}(x)
$$

where $\lambda i$ and $v i$ are both Lagrangian multipliers, and vectors $\lambda$ and $v$ are called dual variables or Lagrangian multiplier vectors. This process actually gives the general construction 


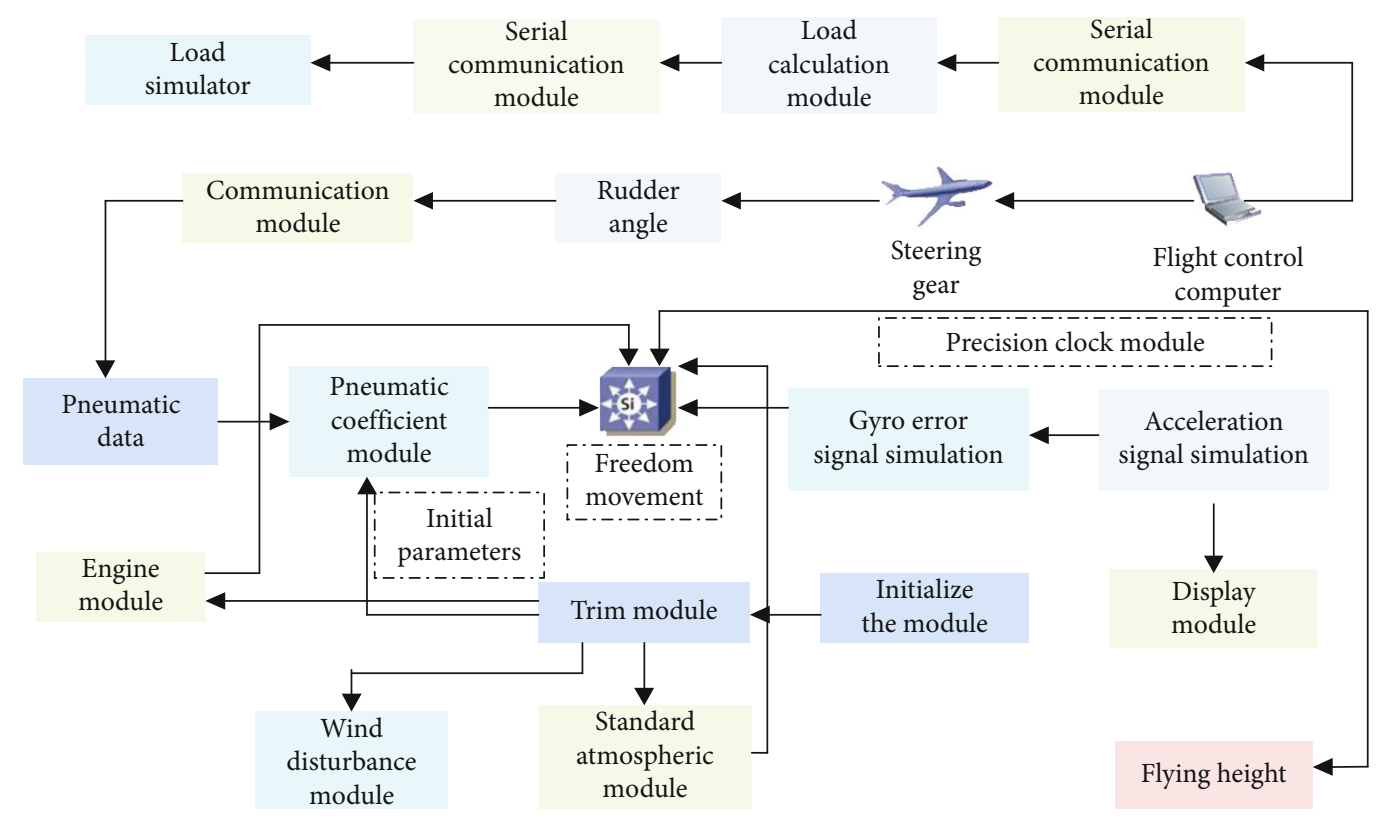

FIGURE 1: UAV flight simulation system block diagram.

method of the Lagrangian function. Further, the Lagrange dual function needs to be defined as follows:

$$
h(v, \lambda)=\inf \left(\sum_{i=1}^{p} v_{i} h_{i}(x)+\sum_{i=1}^{m} f_{i} \lambda_{i}(x)+f_{0}(x)\right)
$$

The value of $x$ in the Lagrangian function defined above should be within the specified range. If the Lagrange function does not specify a lower bound on $x$, then the value of the function may include $-\infty$. It can be seen from the form defined above that the dual function is a piecewise continuous lower bound of a set of affine functions with $(\lambda, v)$ as independent variables, regardless of whether the original optimization problem is a convex function. After transformation, its Lagrangian dual function must be convex.

No matter whether the initial problem is convex or not, the objective function of the Lagrange dual problem is concave, and the constraints are also convex. Therefore, its optimization problem must be a convex optimization problem. According to the theory of convex optimization, the only optimal solution must be obtained, and this result actually guarantees the solvability of the dual optimization problem.

When the optimization of the original problem is transformed into that of its dual problem, the optimization values of the two problems may not be equal. This phenomenon needs to be discussed and analyzed. The next conversion can ensure that the original problem and the dual problem are equal or determine the cause of the difference between the original problem and the dual problem.

2.2. Structural Analysis of UAV-WSN System. The WSN (wireless sensor network) node is usually composed of a microprocessor module, a data storage module, a communication module, a sensor module, and a power supply. The microprocessing module is mainly composed of a bus and an analog-to-digital converter. It is generally used as an IO interface. The storage modules of WSN nodes are generally relatively small, mainly used to store data and programs. The storage module of the WSN node is mainly limited by its size and power consumption. If the WSN node performs read and write operations, the power consumption will increase greatly, so the design of the WSN node must consider the needs of its application. The main modulation methods used by the WSN communication module are amplitude shift keying and frequency shift keying. Because energy consumption is the most prominent problem restricting the use of WSN nodes, when WSN nodes are not communicating, in order to prevent the nodes from wasting too much unnecessary monitoring and achieve energy-saving purposes, WSN nodes are in a dormant state. When the WSN node is activated, it starts to communicate. The WSN sensing module mainly includes temperature sensing, pressure sensing, light sensing, and other methods. The WSN application mainstream sensing module is MEMS (microelectromechanical systems) module. The advantage of MEMS module lies in its small size, low cost, and relatively accurate information about the surrounding environment, which has been widely welcomed by people. The operating system of the WSN node is mainly an embedded operating system, which also has other operating systems. Because the data processed by the WSN node is not very complicated, but its power consumption, size, and storage capacity are strictly limited, so the operating system of the WSN node requires high efficiency and certain fault tolerance. The block diagram of the UAV flight simulation system is shown in Figure 1.

Referring to the TCP/IP network hierarchy model, WSN can also be divided into the physical layer, data link layer, and network layer. Among them, the physical layer and the data link layer are mainly to study how to make the energy more efficient when WSN nodes communicate with each other. 


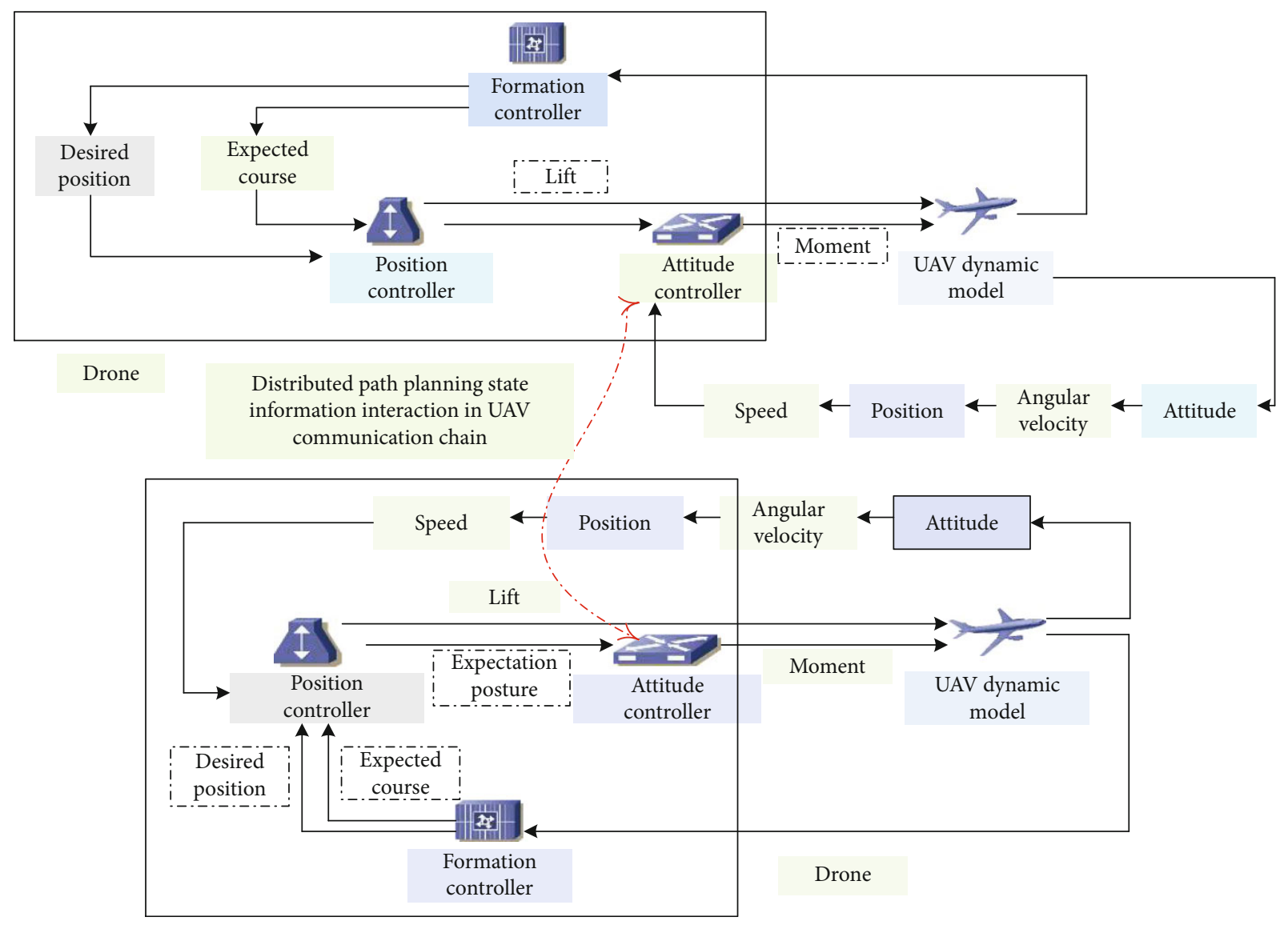

FIGURE 2: UAV formation control structure diagram.

The research on the network layer of WSN mainly focuses on the Routing method. At the network layer, WSN nodes mainly communicate in a multihop manner, and energy has always been one of the most concerned topics in WSN networks. The main research of the network layer is the overall energy of the entire network. If the WSN nodes are fixed, then when collecting information, the information needs to be stored and forwarded through neighboring nodes, which will generate a lot of energy consumption. If mobile nodes are introduced in this network to collect information, UAV is undoubtedly one of the best choices. With UAV's strong maneuverability and sufficient energy supply, it can act as a powerful node with information collection in the network that can move freely and have unlimited energy, which can effectively reduce the energy consumed by information forwarding in the network and extend the entire network.

WSN nodes are mainly randomly deployed in the monitoring area through manual setting or high-altitude broadcasting. WSN nodes form a wireless sensor network mainly through self-organization. The WSN node has the ability to sense, collect, store, and process data. It can process and analyze the information in the monitoring area and can also return the data to the UAV in a multihop manner. The UAV has a relatively strong emission capability and high energy. At the same time, the UAV can also be equipped with a corresponding processor, which has data storage and processing capabilities, can collect data in the entire area, and can transmit data information to the observer. The observer analyzes the received data through the terminal of the network and draws a conclusion to make corresponding changes to the perceived objects. Therefore, the UAV-WSN architecture should include WSN nodes, UAVs, observers, and perceived objects. The structure diagram of UAV formation control is shown in Figure 2.

2.3. Analysis of the Characteristics of UAV-WSN System. UAV-WSN system is mainly composed of WSN nodes in the network. As a sink node, UAV collects information and sends data back to the observer. WSN nodes can also be used as interrupt nodes to route and forward information from WSN nodes that UAV cannot receive. The WSN nodes are connected peer-to-peer.

(1) Large scale and wide range

In order to monitor the situation in a certain area, it is usually necessary to deploy a large number of wireless sensor nodes in the monitoring area. Sometimes the number of wireless sensor nodes may reach thousands or even more. On the one hand, the geographical area of monitoring is particularly broad, and a large number of nodes need to be deployed for monitoring, and then, UAV is used to collect information. For example, deploying WSN nodes in the original forest for monitoring, it is necessary to deploy a large 
number of WSN nodes and, then, use UAV high mobility for information collection. If the space is not very large, but the density requirements of the WSN nodes that need to be deployed are very high, a large number of WSN nodes need to be placed, and UAV can effectively reduce the energy consumed by information forwarding. The advantage of this is that by adjusting the communication distance and angle, the information can have a greater signal-to-noise ratio, and the distributed processing of a large amount of collected information can also improve the accuracy of monitoring and improve the fault tolerance of the entire system. The high dependence on the sensor of a node makes the whole system in a normal working state; the presence of a large number of redundant nodes, even if an individual node fails, will not greatly affect the entire system, which also makes the system very strong fault-tolerant performance; when a large number of nodes cover the monitoring area, the monitoring area is in full coverage, which effectively reduces blind spots and improves the monitoring capacity of the entire system. The schematic diagram of the UAV positioning system is shown in Figure 3.

\section{(2) Dynamic network}

The WSN topology is not fixed and may change due to the following factors: first, the sensor node malfunctions or fails due to the harsh environment of the wireless sensor location or the battery exhaustion; second, the changes in environmental conditions may affect the wireless. The mutual communication bandwidth between sensors affects the quality of communication, and when it is disconnected, it makes the topology of the entire WSN network unstable; third, relative to a fixed wireless sensor, if the sensor can be moved, which brings uncertainty to the entire system, then the network structure will also change with the changes of the members in the system; fourth, when new wireless sensor nodes are added in the network, then the existing network must be reorganized to form a new topology structure, which also shows that the wireless sensor network has dynamic system reconfigurability.

Because UAV can move arbitrarily, the network topology is also changing at any time. This change is not only limited to the communication between UAV and WSN but also related to the transmission of information between WSN nodes. Because the communication bandwidth of UAV is fixed, within a certain range, the number of communication received by WSN nodes is also very limited, which requires that the information between WSN nodes needs to be collected and sent to UAV.

\section{UAV Global Path Planning Algorithm Based on the Ant Colony Algorithm}

3.1. The Ant Colony Algorithm. The process of ants searching for food sources can be described as follows: in the absence of pheromone information, individual ants randomly select one of the paths when they pass the intersection and return to the original path after finding the food source. In this process, a large number of ants will leave more pheromones along the same path. Therefore, the probability of subsequent ants choosing a short path will be relatively large. As more and more ants choose a shorter path, the concentration of pheromones on this path will continue to increase, and because pheromones are a volatile substance, the pheromone on other paths will gradually dissipate with the passage of time, and eventually, all ants will choose the shortest path forward. This collective behavior of the ant colony exhibits a selfcatalytic enhancement effect: the more ants choose a path, the more attractive this path will be for subsequent ants, so a positive feedback is formed. Positive feedback ultimately selects the shortest foraging path. Figure 4 shows the process of ants finding the shortest foraging path in an obstacle environment.

In the ant colony algorithm, the concept of artificial ants was abstracted according to the ants in the biological world, and some key ant behavior characteristics in the ant colony foraging process were given to artificial ants. Worker ants have some characteristics that real ants do not have. Each artificial ant is a simple individual satisfying the following behavioral characteristics:

(1) The movement of artificial ants is a transition between two discrete states

(2) The artificial ant moves along the adjacent position. When it is at any position, it selects the next visited position according to a certain probability. This probability is related to the pheromone concentration and distance between the two positions

(3) Artificial ants release pheromones along the way during movement

(4) The artificial ant has a memory function, and it will not select the location that has been visited during a pathfinding process

In the ant volume system, the pheromone increment is inversely proportional to the length of the path, so that more pheromone is obtained on the shorter path, thereby generating greater attraction for subsequent ants.

The ant-week system model releases pheromones after the ants have established a complete trajectory. The pheromone updating formula is as follows:

$$
\begin{aligned}
\tau_{i j}(n+t) & =\Delta \tau_{i j}(n+t, t)+(1+\rho) \times \tau_{i j}(t), \\
\Delta \tau_{i j}(t+n, t) & =\sum_{k=0}^{m} \Delta \tau_{i j}^{k}(t+n, t),
\end{aligned}
$$

where $p$ is the pheromone volatilization coefficient. The addition of volatility coefficient can prevent the algorithm from premature convergence to a suboptimal solution to a certain extent, so as to ensure that the algorithm still has a probability to explore new areas in the space after iterating for a period of time. $\Delta \tau_{i j}^{k}(t+1, t)$ represents the amount of pheromone released by the $k$ th ant on the branch $(i, j)$.

The process of the ants searching for the shortest path from the ant nest to the food source is the result of the 


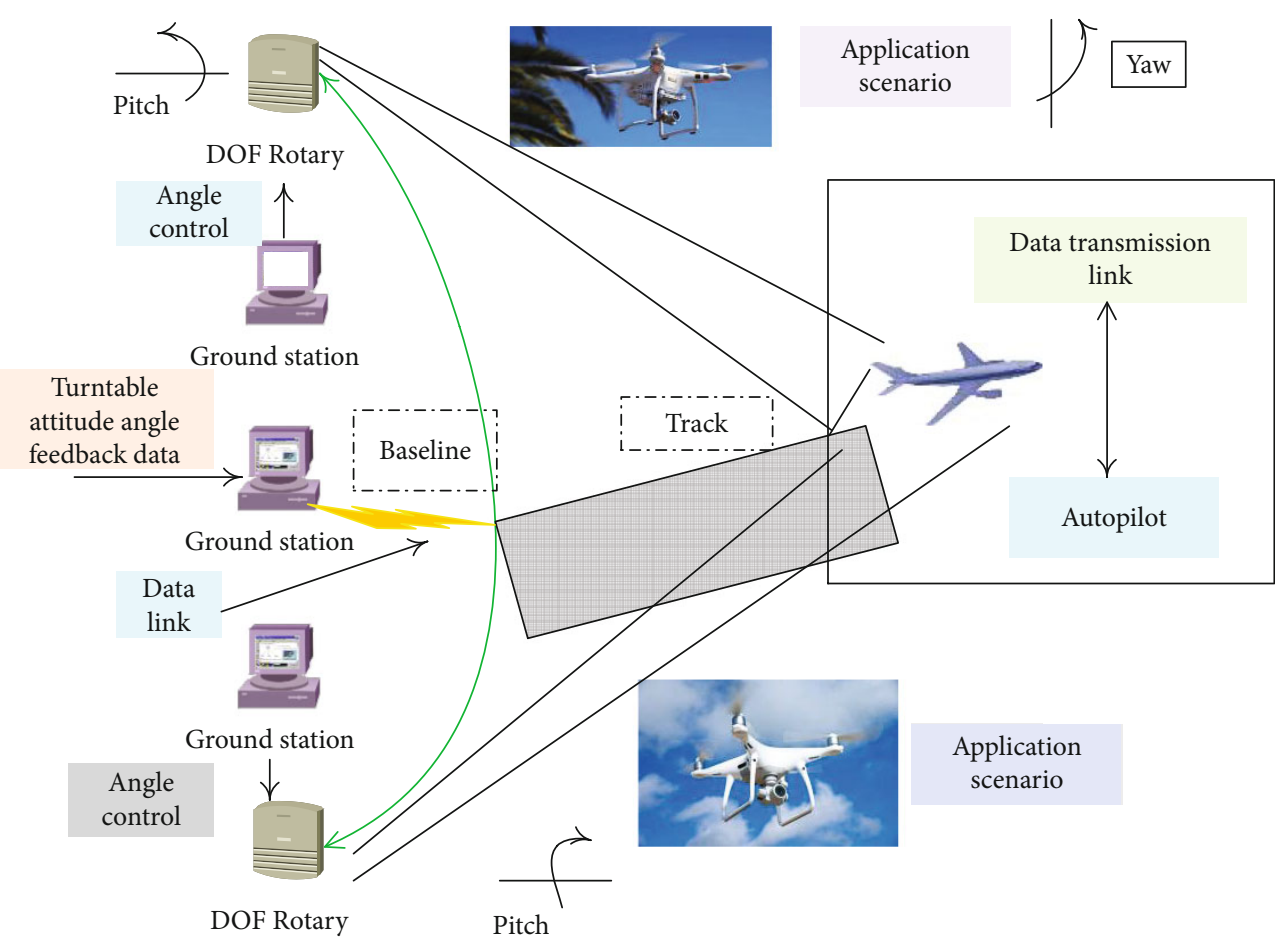

FIGURE 3: Schematic diagram of UAV positioning system.

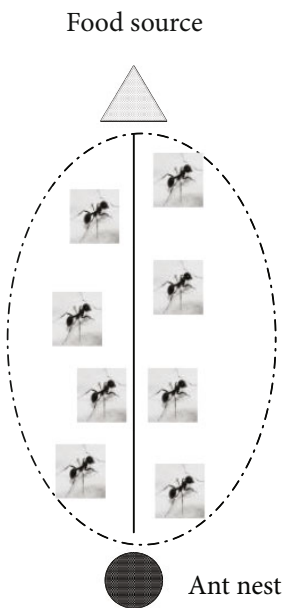

Travel normally
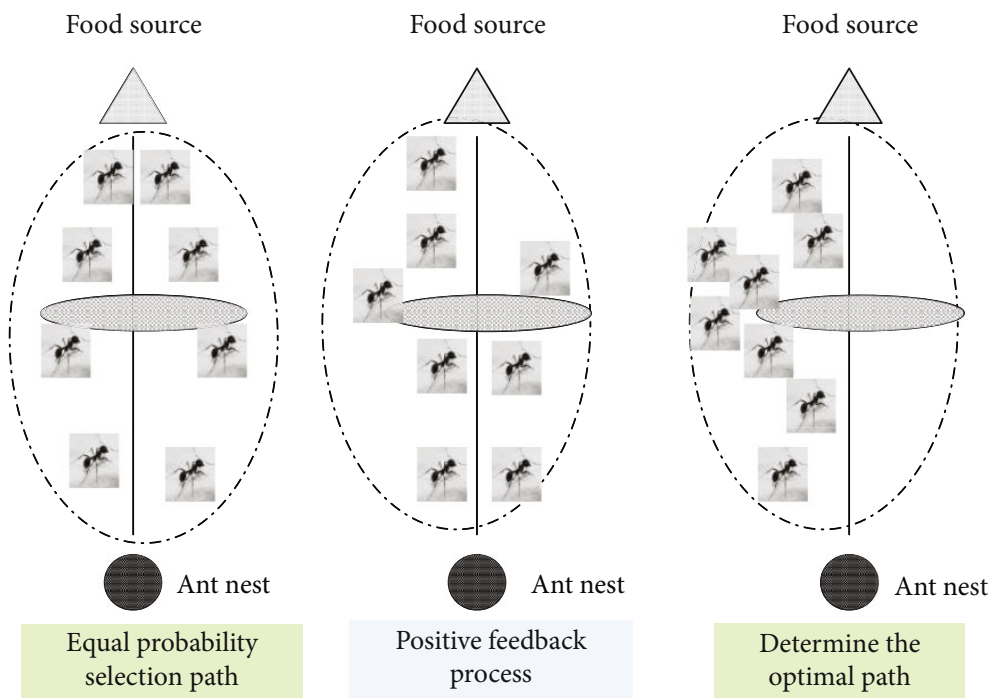

FIGURE 4: Schematic diagram of ant colony searching for food source.

interconnection, mutual influence, interaction, and mutual cooperation between a large number of ant individuals. The ant colony algorithm uses artificial ants to simulate the path-finding behavior of ants, which is essentially characterized by positive feedback and good parallelism. At the same time, as an intelligent bionic algorithm, the ant colony algorithm has very good optimization capabilities. The performance of the solution of the ant colony algorithm increases with the increase of the number of ants, until it reaches the upper limit, that is, the ideal situation that can always find the optimal solution. In addition, through reasonable improvements in parameter settings, probability calculation heuristics, and pheromone update strategies, the algorithm can also be improved in terms of convergence speed and search capabilities.

However, the ant colony algorithm itself is a probabilistic algorithm, and it is difficult to mathematically prove its correctness and reliability systematically. When the ant colony algorithm solves the optimization problem, the high-level search behavior of the algorithm is generated by the mutual influence of the simple behavior of the underlying ants. The design of the search algorithm for a single ant is simple, but the control of the entire system is not easy. The designer must be able to map the complex optimization behavior of the 
entire system to the simple behavior of the individual ants at the bottom and achieve the desired by controlling the bottom search process. High-level behavioral characteristics are more difficult.

3.2. UAV Path Planning Algorithm Design Based on the Ant Colony Algorithm. The ant colony algorithm has the advantages of positive information feedback, good parallelism, and optimization capabilities, but it still has some shortcomings. For example, due to the lack of pheromones in the early stage, the movement of the ant colony lacks guidance information, and the solution speed is slow. With the effect of feedback, the algorithm is prone to fall into local extreme value. In view of the shortcomings of the basic ant colony algorithm and the specific application object of UAV path planning, this article made some adjustments and improvements to the basic ant colony algorithm.

The ant colony algorithm uses the evolutionary process of the colony to search for the optimal solution. A single ant builds a feasible solution and adds it to the feasible solution set after a pathfinding process. The solution constructed by $m$ ants after a cycle forms a subset of the feasible solution set. Therefore, the larger the number of ants, the larger the subset constructed, the stronger the global search ability of the ant colony algorithm, and the better the stability of the algorithm. In the actual application of the algorithm, if all ants are set to release pheromones, when the number of ants is too large, although the randomness of the search is strengthened, the change of pheromones on each edge tends to be average, which weakens the positive feedback effect of information and reduces the convergence speed. In this paper, we have improved the pheromone global update mode to only release pheromones by the iterative optimal ants, and there is no problem that a large number of ants weaken the positive feedback. However, the increase in the number of ant population will inevitably lead to the increase in the time complexity of the algorithm, and the time spent in each iteration will increase. On the contrary, if the number of ants is too small, the subset of the problem constructed is too small, the randomness of the search path is weakened, and the global optimal solution may not be found when the size of the problem is large. Therefore, the determination of the number of ants is a balance between the speed of convergence and the searching ability.

According to the definition of current visibility, the visibility value of a candidate grid is only related to its distance from the current grid. In this way, there is a problem: when the ants are in any grid, the ants will always prefer to select the grid with the smallest distance from the current grid, regardless of the grid and the target point. When the initial pheromone concentration is low and it is impossible to give enough guidance information for the ant's path selection, the ant selects the grids in these directions with almost the same probability to start the search. Due to the lack of information related to the distance of the destination as a guiding factor, the ant's path search is aimless, and it often takes a large circle in the environment map to find the destination, which not only results in a slow initial search speed of the algorithm but also a low search ability. It is difficult to search for the true optimal path.

In response to the above problem, the visibility from grid $r$ to grid $s$ in the transition probability calculation formula is changed to the visibility between the candidate grid and the target grid. After adopting this strategy, the ants no longer blindly conduct path search, but preferentially select the grid closest to the target point in the candidate grid set, so this strategy is called the target attraction strategy.

In the ant colony algorithm, each ant individual uses a greedy heuristic to perform a path search. Each ant can find a feasible solution. The ant individuals in the ant colony have established a large number of solutions at the same time. The higher-quality solutions are rewarded, and the pheromone on its path is more strengthened, so that through continuous positive feedback, the solution of the problem gradually evolves toward the direction of global optimality until the optimal solution is found.

The above is the principle of the optimal solution of the ant colony algorithm in the ideal case. However, in fact, the ant colony algorithm does not always find the global optimal solution. In some cases, the positive feedback of the system may lead to premature convergence of the solution. For example, because there is a local optimal solution in the system, or just because of random oscillations in the early iterations, some nonoptimal individuals in the group affect the entire group, preventing the group from further exploring the global optimal solution.

After adopting the goal attraction strategy and defining the visibility with the reciprocal of the distance between the candidate grid and the target grid, the premature convergence problem will appear in some specific cases: when there is a local optimal solution in the algorithm, this solution is global; when the path length difference of the optimal solution is not large, the path of the ant colony will converge to this local optimal solution.

This article proposes a new global pheromone update rule as follows:

$$
\tau_{r s}(t+n)=\Delta \tau_{r s}(t, t+n) \times \rho+(1+\rho) \times \tau_{r s}(t+1) .
$$

In the optimization process of the ant colony algorithm, with the enhancement of positive feedback, the pheromone on the edge of the optimal solution (it may also be actually a suboptimal solution) continues to accumulate, and the pheromone on the other edge continuously volatilizes. The gap is getting bigger and bigger, and the ants will repeatedly construct the same solution, search stagnation, and no longer explore new solutions. Therefore, slowing down the increasing speed of the pheromone gap between the edge searched by more ants and the edge rarely searched can ensure that the system evolves at a reasonable speed, avoiding the premature convergence phenomenon caused by the positive feedback speed being too fast appear.

In the ant's path search process, sometimes, there is no way to go after the ant has reached a certain position, that is, the candidate grid set allowed is an empty set, the algorithm is stuck in a deadlock state, and the path search is stalled. Special consideration should be given to this 


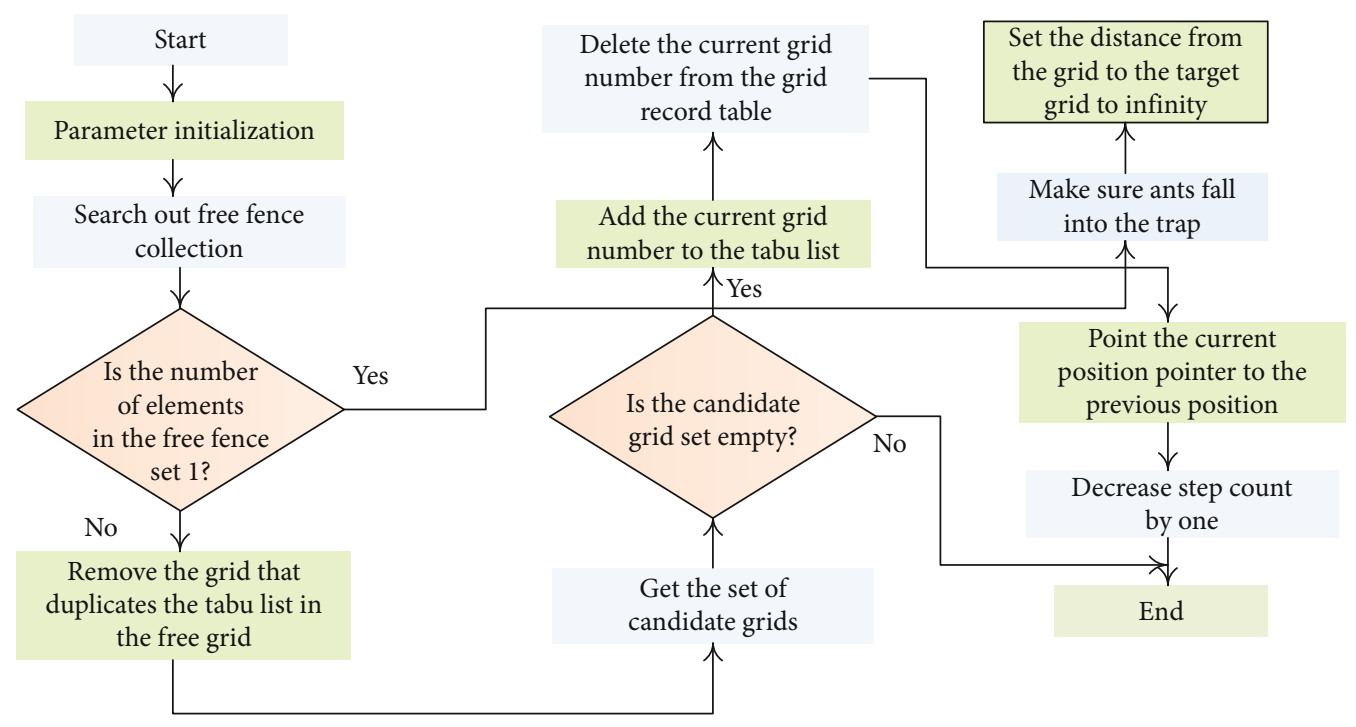

FIgURE 5: Flow chart of path rollback strategy.

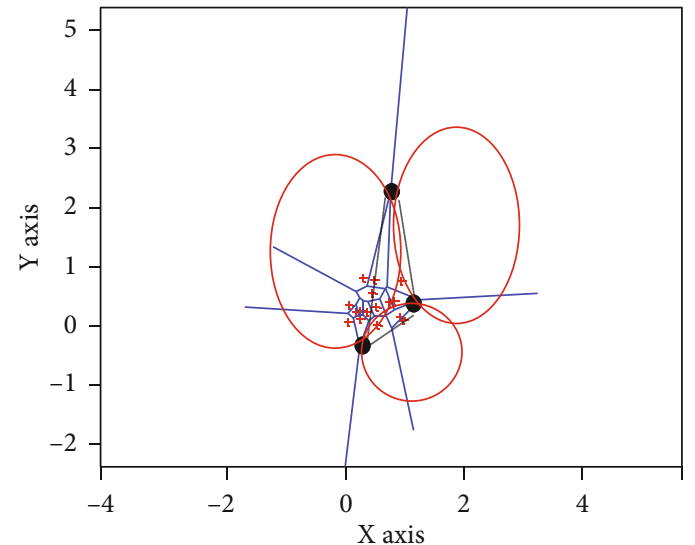

(a)

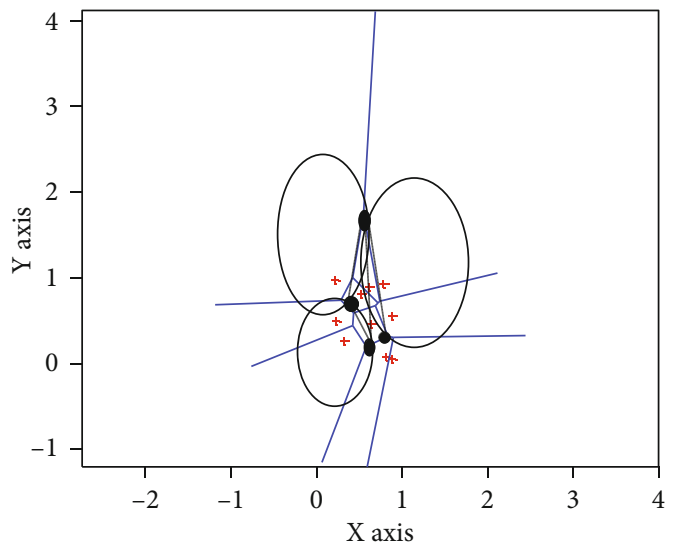

(b)

Figure 6: Voronoi diagram construction results. (a) Voronoi diagram construction results of the original dual generation method. (b) Voronoi diagram construction results of the ant colony dual generation method.

situation, and a certain backoff strategy should be set to make the algorithm adapt to various complex environments and improve the robustness of the algorithm. The flow chart of the path rollback strategy is shown in Figure 5.

\section{Simulation and Discussion}

4.1. Simulation of the Method of Generating V Map. This paper mainly studies the obstacle avoidance of multiquadrotor UAV formation on the basis of formation control constraints. In the obstacle avoidance research, it involves the global flight path planning and local obstacle avoidance. Then, the Dijkstra algorithm and $\mathrm{A}^{*}$ algorithm are introduced and compared. Finally, $\mathrm{A}^{*}$ algorithm is used to simulate the global path planning of multi-UAV formation.

In order to test whether the ant colony duality generation method can obtain a more secure V-graph path, this section simulates the original duality generation method and the ant colony duality generation method under the abovementioned simulation test conditions and compares them.

Figure 6(a) is the simulation result of the original dual generation method under the given obstacle environment. Among them, the black dot is the center of the obstacle safety circle, the black dotted line is the Delaunay triangulation generated by the duality generation method, the red circle is the circumscribed circle of each triangle in the Delaunay triangulation, and the solid black line is the Voronoi diagram finally obtained path results.

Figure 6(b) shows the simulation results of the ant colony dual generation method in the obstacle environment. Among them, the black dot is the center of the safety circle of the obstacle, the black dotted line is the Delaunay triangulation generated by using the even generation method with the center of the safety circle as the vertex, the black circle is the circle tangent to the three adjacent safety circles, and the solid blue line is the final Voronoi diagram path result. 


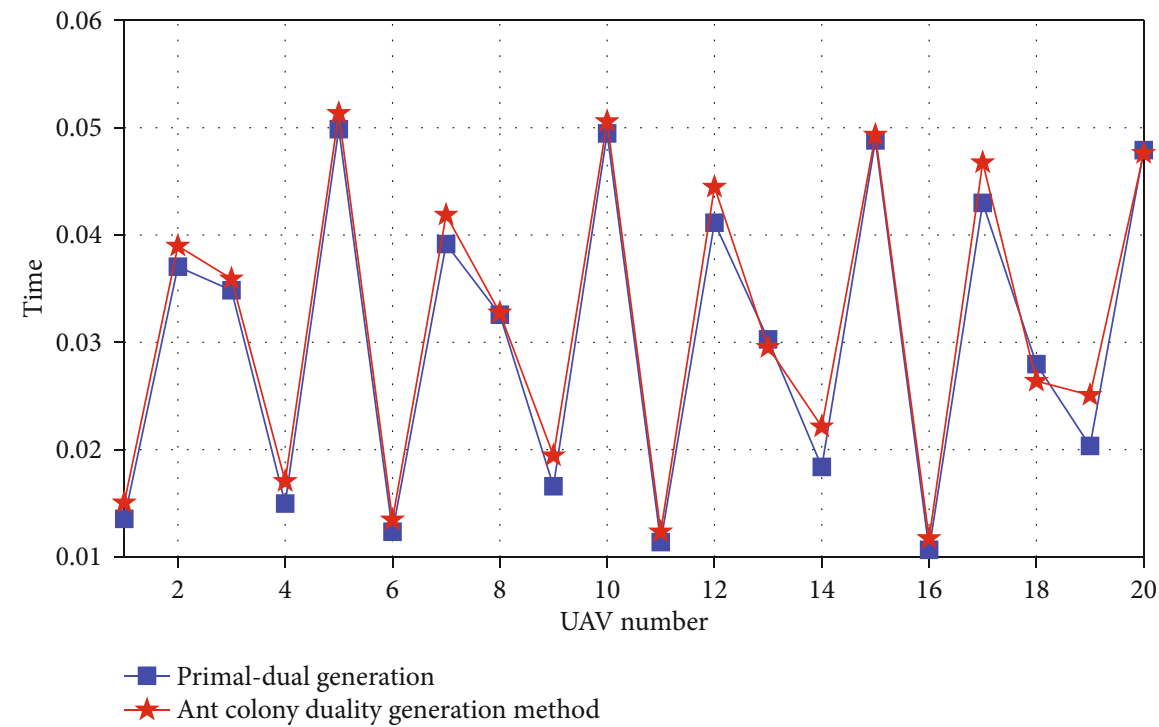

FIGURE 7: Comparison of time before and after improvement of the duality generation method.

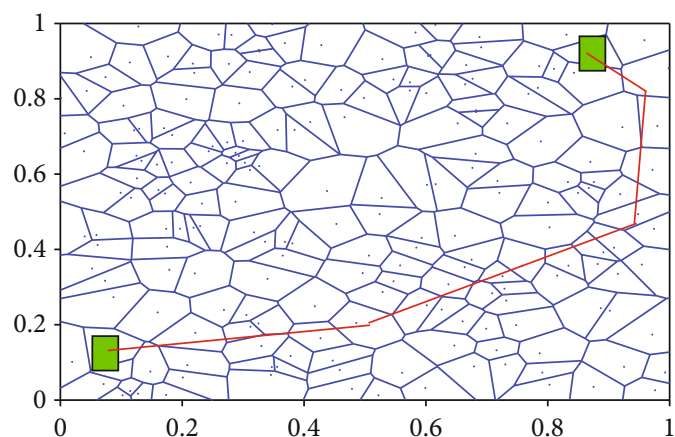

(a)

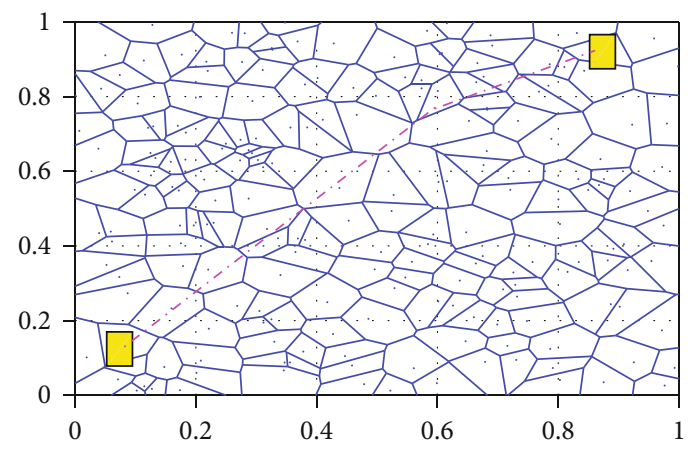

(b)

Figure 8: Comparison of simulation results in a multidimensional environment. (a) The Dijkstra algorithm. (b) The dual decomposition ant colony algorithm.

From the experimental results, it can be seen that although the path maps obtained by the two methods are not very different overall, the original dual generation method produced a path that intersects the safety circle of the obstacle. It can be seen from the figure that compared with the original dual generation method, the improved dual generation method generates a Voronoi diagram with an equal safety circle distance to the obstacle, making the obtained Voronoi diagram path safer.

Figure 7 shows the time used by the two methods in the above experiment. It can be seen that the ant colony duality generation method is not as good as the original duality generation method in terms of time performance. But in terms of UAV offline path planning, there is enough time to do this path planning, the time requirement is not very high, and UAV has a certain flying height; at this height, the amount and scale of obstacles are usually not very large, so the improved dual generation method can get a safer Voronoi diagram path under certain time conditions.

4.2. Simulation Comparison between the Dijkstra Algorithm and Dual Decomposition Ant Colony Algorithm. In order to compare the performance of the Dijkstra algorithm and dual decomposition ant colony algorithm in global path planning, this section selects multidimensional simple environment with one obstacle by analyzing the predecessors' setting of the number of obstacles in the path planning simulation. Simulation experiments were carried out on these two environments under the abovementioned simulation experiment conditions.

Because the simulation of directly inputting the Voronoi diagram is difficult to visually observe, so when doing the simulation, this article will rasterize the $\mathrm{V}$ diagram, the center of each square is the vertex of the Voronoi diagram, and the connection between the centers is the $\mathrm{V}$ diagram. The simulation effect is shown below.

Figure $8(a)$ is the simulation result of the Dijkstra algorithm, and Figure $8(\mathrm{~b})$ is the simulation result of dual decomposition ant colony algorithm. It can be seen that in this example, the Dijkstra algorithm accesses more nodes than the dual decomposition ant colony algorithm, and in the results obtained, the dual decomposition ant colony algorithm finds more paths than the Dijkstra algorithm finds. 


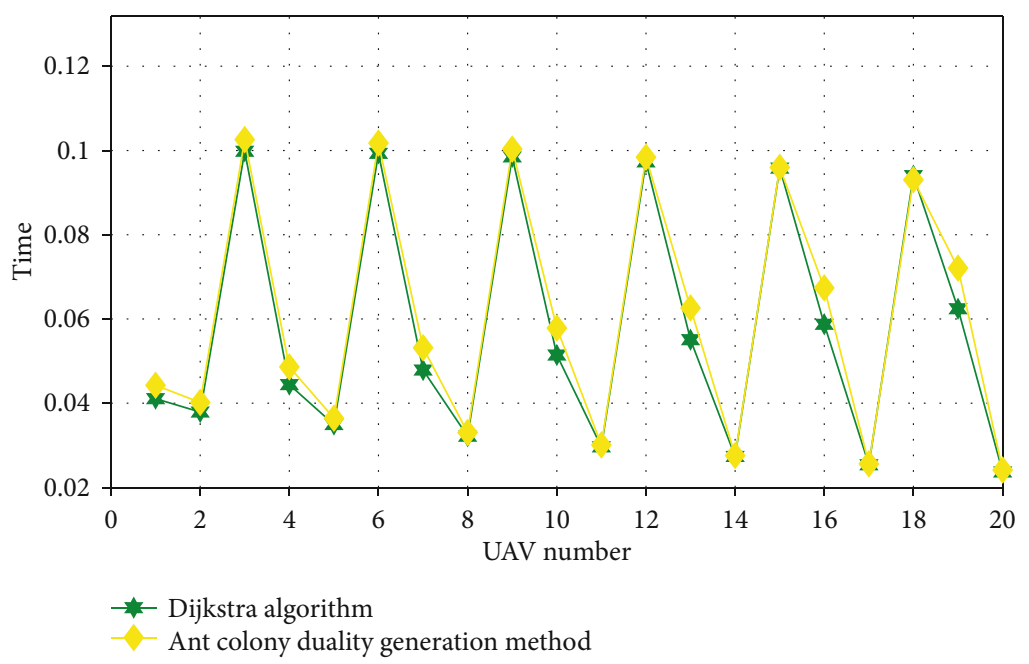

FIGURE 9: Comparison of time between the dual decomposition ant colony algorithm and Dijkstra algorithm.

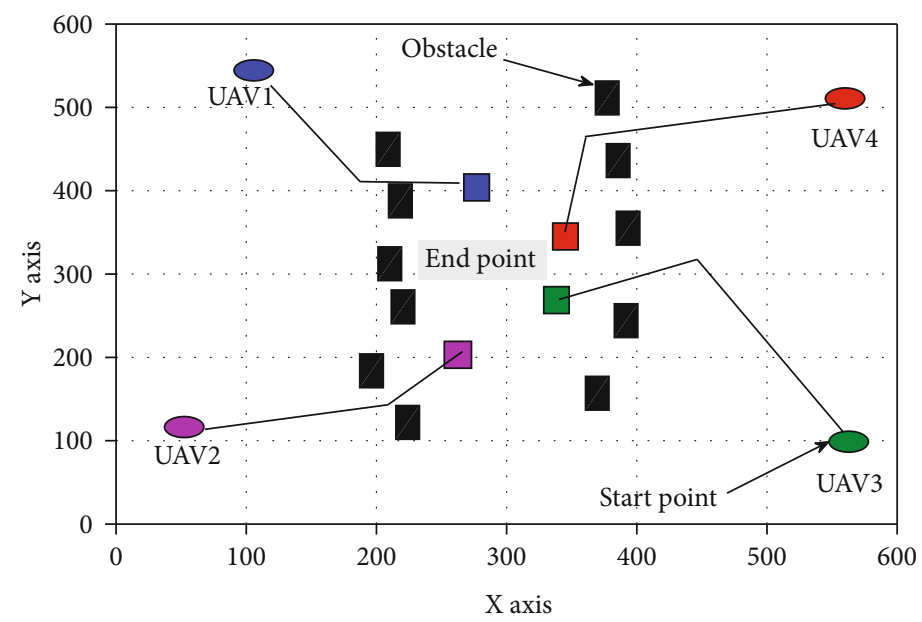

(a)

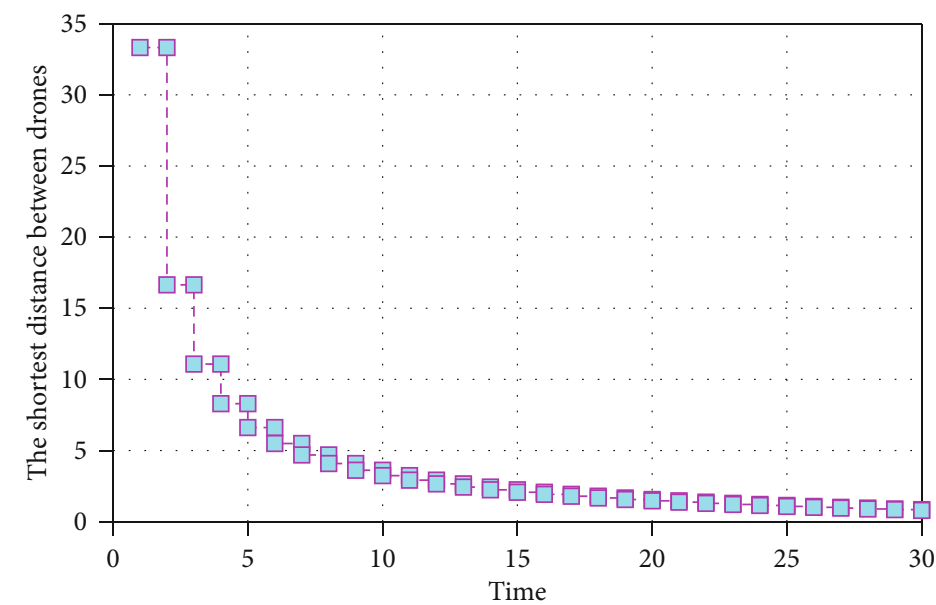

(b)

FIGURE 10: The results of UAV distributed path planning under dual decomposition ant colony algorithm. (a) Planned trajectory results. (b) Curve of the shortest distance between drones. 
Figure 9 shows the average program elapsed time calculated by running the Dijkstra algorithm and the dual decomposition ant colony algorithm ten times in this simulation experiment. It can be seen from Figure 9 that the dual decomposition ant colony algorithm is more efficient than the Dijkstra algorithm in terms of its own time.

Through the simulation comparison of the Dijkstra algorithm and dual decomposition ant colony algorithm in a relatively complex obstacle environment of 20 dimensions, it is concluded that the dual decomposition ant colony algorithm is superior to the Dijkstra algorithm in path optimization and time consumption. The ant colony algorithm is more efficient. Therefore, this paper chooses the dual decomposition ant colony algorithm to realize the global path planning for the UAV cluster.

4.3. The Dual Decomposition Ant Colony Algorithm to Find the Optimal Path of the Cluster Simulation. This section will use dual decomposition ant colony algorithm to simulate multi-UAV formation path planning based on application scenarios and formation rules.

Considering the two typical tasks of UAV formation assembly and formation reconstruction, the effectiveness of the sequential convex optimization method to solve the collaborative trajectory planning problem is verified. Formation assembly requires that the drones in the formation arrive at the designated assembly target point from their respective initial positions at the same time and form the desired formation, which provides conditions for the formation to perform subsequent collaborative tasks. Formation reconstruction requires that the UAV formation change its formation through coordinated flight to meet the mission or safety needs.

It can be seen from Figure 10(a) that the trajectory obtained by the dual decomposition ant colony algorithm planning method can realize the transfer from the starting point to the target point and avoid obstacles in the environment. The roundabout approach guarantees the coordination of the entire formation time. According to the variation curve of the shortest distance between drones in Figure 10(b), the shortest distance between formations is always maintained above the safe distance, that is, the planned trajectory of the UAV formations can meet the collision avoidance constraints. Therefore, the dual decomposition ant colony algorithm can plan a feasible cooperative trajectory that satisfies the constraints for the UAV formation.

\section{Conclusions}

In this paper, we use the popular ant colony algorithm in the path planning field to study the indoor UAV global path planning problem; theoretically analyze the characteristics, advantages, and disadvantages of the ant colony algorithm in detail; and propose a distributed UAV communication chain path planning algorithm. The algorithm improves the basic ant colony algorithm in path selection strategy, pheromone update, fallback strategy, etc., and enhances the algorithm's adaptability to the environment and the algorithm's robustness, making the algorithm's ability to achieve the best condition. The ant colony duality generation method is compared with the original duality generation method, and the improved method can obtain a safer flight path Voronoi diagram under a certain time limit. In a simple environment with one obstacle and a complex environment with multiple obstacles, the simulation comparison between Dijkstra's algorithm and the dual decomposition ant colony algorithm is obtained, and the dual decomposition ant colony algorithm is obtained in the path optimization and time consumption. Both are superior to the Dijkstra algorithm, and the dual decomposition ant colony algorithm is more efficient. To verify the effectiveness of the dual decomposition ant colony algorithm in multi-UAV path planning, in the application scenario proposed in the previous chapter (under the environment of four obstacles and four UAVs), the dual decomposition ant colony algorithm is used. The four-rotor UAV formation flight has been path-planned, and it is concluded that the dual decomposition ant colony algorithm can plan a safe flight path that conforms to the multirotor UAV formation rules. The real-time programming algorithm in this paper is designed based on the assumption that the sensor detection range can only support one-step programming. In future research, an online planning algorithm can be designed according to the actual detection range of the sensor, which can be used for path optimization within a certain range.

\section{Data Availability}

No data support is available.

\section{Consent}

Informed consent was obtained from all individual participants included in the study references.

\section{Conflicts of Interest}

We declare that there is no conflict of interest.

\section{Acknowledgments}

This study was supported by the Scientific Research Project of Zhejiang Provincial Education Department (No: Y201839845), Science and Technology Plan Projects of Quzhou (No: 2018k12).

\section{References}

[1] H. Van Nguyen, H. Rezatofighi, B. N. Vo, and D. C. Ranasinghe, "Online UAV path planning for joint detection and tracking of multiple radio-tagged objects," IEEE Transactions on Signal Processing, vol. 67, no. 20, pp. 5365-5379, 2019.

[2] T. I. Zohdi, "The game of drones: rapid agent-based machinelearning models for multi-UAV path planning," Computational Mechanics, vol. 65, no. 1, pp. 217-228, 2020.

[3] K. Y. Kok and P. Rajendran, "Enhanced particle swarm optimization for path planning of unmanned aerial vehicles," 
ECTI Transactions on Computer and Information Technology (ECTI-CIT), vol. 14, no. 1, pp. 67-78, 2020.

[4] S. Primatesta, G. Guglieri, and A. Rizzo, "A risk-aware path planning strategy for uavs in urban environments," Journal of Intelligent \& Robotic Systems, vol. 95, no. 2, pp. 629-643, 2019.

[5] H. Wang, J. Wang, G. Ding, J. Chen, F. Gao, and Z. Han, "Completion time minimization with path planning for fixed-wing UAV communications," IEEE Transactions on Wireless Communications, vol. 18, no. 7, pp. 3485-3499, 2019.

[6] X. Peng, Y. Lan, J. Hu, F. Ouyang, K. Xiao, and Z. Gao, “Turning mode and whole region-coverage path planning and optimization of agricultural small UAV," Journal of South China Agricultural University, vol. 40, no. 2, pp. 111-117, 2019.

[7] M. d. S. Arantes, C. F. M. Toledo, B. C. Williams, and M. Ono, "Collision-free encoding for chance-constrained nonconvex path planning," IEEE Transactions on Robotics, vol. 35, no. 2, pp. 433-448, 2019.

[8] G. Zhang and L. T. Hsu, "A new path planning algorithm using a GNSS localization error map for UAVs in an urban area," Journal of Intelligent \& Robotic Systems, vol. 94, no. 1, pp. 219-235, 2019.

[9] Y. Ding, B. Xin, and J. Chen, "Precedence-constrained path planning of messenger UAV for air-ground coordination," Control Theory and Technology, vol. 17, no. 1, pp. 13-23, 2019.

[10] R. K. Dewangan, A. Shukla, and W. W. Godfrey, "Three dimensional path planning using Grey wolf optimizer for UAVs," Applied Intelligence, vol. 49, no. 6, pp. 2201-2217, 2019.

[11] G. A. Thanellas, V. C. Moulianitis, and N. A. Aspragathos, "A spatially wind aware quadcopter (UAV) path planning approach," IFAC-Papers OnLine, vol. 52, no. 8, pp. 283-288, 2019.

[12] Y. Qian, F. Wang, J. Li, L. Shi, K. Cai, and F. Shu, "User association and path planning for UAV-aided mobile edge computing with energy restriction," IEEE Wireless Communications Letters, vol. 8, no. 5, pp. 1312-1315, 2019.

[13] Y. Choi, Y. Choi, S. Briceno, and D. N. Mavris, "Energy-constrained multi-UAV coverage path planning for an aerial imagery mission using column generation," Journal of Intelligent \& Robotic Systems, vol. 97, no. 1, pp. 125-139, 2020.

[14] S. Cho and D. H. Shim, "Sampling-based visual path planning framework for a multirotor uav," International Journal of Aeronautical and Space Sciences, vol. 20, no. 3, pp. 732-760, 2019.

[15] U. Challita, W. Saad, and C. Bettstetter, "Interference management for cellular-connected UAVs: a deep reinforcement learning approach," IEEE Transactions on Wireless Communications, vol. 18, no. 4, pp. 2125-2140, 2019.

[16] V. Jamshidi, V. Nekoukar, and M. H. Refan, "Analysis of parallel genetic algorithm and parallel particle swarm optimization algorithm UAV path planning on controller area network," Journal of Control, Automation and Electrical Systems, vol. 31, no. 1, pp. 129-140, 2019.

[17] U. Challita, A. Ferdowsi, M. Chen, and W. Saad, "Machine learning for wireless connectivity and security of cellularconnected UAVs," IEEE Wireless Communications, vol. 26, no. 1, pp. 28-35, 2019.

[18] P. Oettershagen, J. Förster, L. Wirth, G. Hitz, R. Siegwart, and J. Ambühl, "Meteorology-aware multi-goal path planning for large-scale inspection missions with solar-powered aircraft,"
Journal of Aerospace Information Systems, vol. 16, no. 10, pp. 390-408, 2017.

[19] S. Kumar Debnath, R. Omar, N. B. Abdul Latip et al., "A review on graph search algorithms for optimal energy efficient path planning for an unmanned air vehicle," Indonesian Journal of Electrical Engineering and Computer Science, vol. 15, no. 2, pp. 743-749, 2019.

[20] C. Liu, S. Zhang, and A. Akbar, "Ground feature oriented path planning for unmanned aerial vehicle mapping," IEEE Journal of Selected Topics in Applied Earth Observations and Remote Sensing, vol. 12, no. 4, pp. 1175-1187, 2019. 